\title{
Enhancing sensitivity of SERRS nanoprobes by modifying heptamethine cyanine-based reporter molecules
}

\author{
Yunfei Zhang*, Danqi Li†, Xingyu Zhou*, Xihui Gao*, \\ Shengyuan Zhao* and Cong $\mathrm{Li}^{*}, *$ \\ *Key Laboratory of Smart Drug Delivery \\ Ministry of Education \& PLA, School of Pharmacy \\ Fudan University, 826 Zhangheng Rd., Shanghai, 201203, P. R. China \\ ${ }^{\dagger}$ First Clinical College of Tongji Medical College \\ Huazhong University of Science and Technology \\ 13 Hangkong Rd., Wuhan, Hubei, 430030, P. R. China \\ *congli@fudan.edu.cn
}

Received 22 April 2016

Accepted 18 May 2016

Published 21 June 2016

\begin{abstract}
Surface enhanced resonance Raman scattering (SERRS) is a physical phenomenon that occurs when the energy of incident light is close to that of electronic excitation of reporter molecules (RMs) attached on substrates. SERRS has showed great promise in healthcare applications such as tumor diagnosis, image-guided tumor surgery and real-time evaluation of therapeutic response due to its ultra-sensitivity, manipulating convenience and easy accessibility. As the most widely used organic near-infrared (NIR) fluorophore, heptamethine cyanines possess the electronic excitation energy that is close to the plasmon absorption energy of the gold nano-scaffolds, which results in the extraordinary enhancement of the SERRS signal. However, the effect of heptamethine cyanine structure and the gold nanoparticle morphology to the SERRS intensity are barely investigated. This work developed a series of SERRS nanoprobes in which two heptamethine cyanine derivatives (IR783 and IR780) were used as the RM and three gold nanoparticles (nanorod, nanosphere and nanostar) were used as the substrates. Interestingly, even though IR780 and IR783 possess very similar chemical structure, SERRS signal produced by IR780 was determined as 14 times higher than that of IR783 when the RM concentration was $6.5 \times 10^{-6} \mathrm{M}$. In contrast, less than 4.0 fold SERRS signal intensity increase was measured by changing the substrate morphologies. Above experimental results indicate that finely tuning the chemical structure of the heptamethine cyanine could be a feasible way to develop robust SERRS probes to visualize tumor or guide tumor resection with high sensitivity and target to background ratio.
\end{abstract}

\$Corresponding author.

This is an Open Access article published by World Scientific Publishing Company. It is distributed under the terms of the Creative Commons Attribution 4.0 (CC-BY) License. Further distribution of this work is permitted, provided the original work is properly cited. 
Keywords: Surface enhanced resonance Raman scattering; gold nanoparticles; reporter molecules; heptamethine cyanine.

\section{Introduction}

Surface enhanced Raman scattering (SERS) was first discovered by Fleishmann in 1974. When the pyridine absorbed on roughened silver electrode, it exhibited uncanny enhancement of Raman intensities $\left(10^{6}\right.$ times $){ }^{1}$ As enhancement factor $(\mathrm{EF})$ of Raman signal is proportional to electric field in surface of substrate and polarizability which respectively correspond to electromagnetic (EM) enhancement and chemical (CM) enhancement, ${ }^{2,3}$ it is generally accepted that EM and CM mainly contribute to SERS together. Due to the ultra-sensitivity and sharp fingerprint-like spectra, SERS has showed great potential in diagnosis of tumor. ${ }^{4,5}$ Multiple SERS probes have been exploited and achieved high level of specificity and diagnostic sensitivity. ${ }^{6-8}$ Tumor visualization and image guided completed excision of tumor such as malignant brain tumor have greatly fascinated investigators. ${ }^{9,10}$ Interestingly, recent studies showed the feasibility to accurately excise tumor under the guidance of SERS. Karabeber et al. have achieved the goal of accurately outlining the margin of the glioblastoma and resecting the brain tumor in the animal model. ${ }^{11}$ Therefore, it is obvious that SERS has enormous potential for clinical translation in the field of diagnosis and treatment of tumor. However, there still exits some limitations and disadvantages for SERS such as poor reproducibility, low stability and limited applicability. Above all, the most critical challenge is the synthesis of SERS probes with robust signal for clinical applications. To overcome above problem, two approaches have been done to enhance the Raman signal: (1) Development of novel metallic substrates with high signal enhancement, high stability and good reproducibility. For example, metallic substrates such as silver, ${ }^{12}$ gold, ${ }^{13}$ and copper $^{14}$ nanoparticles have been developed. However, the most pervasive choice is gold as it possesses inert chemical properties, good biocompatibility and the convenience to adjust the size and morphology. Moreover, it has been documented that nanoscale tip and spatial proximity of gold nanoparticle could result in "hot spot" effect, which would significantly increase Raman signal due to the huge increase of local electric field. ${ }^{15}$ Hence, endeavors were put forward in developing gold nanoparticles of different morphologies including nanostar, ${ }^{16}$ nanosphere,${ }^{17}$ nanoporpcorn, ${ }^{18}$ nanoflower, ${ }^{19}$ nanorod ${ }^{20}$ nanocage ${ }^{21}$ and so on. (2) Development of RM that generates Raman signal with high intensity and sharp fingerprint-like peak after it is labeled on the substrates. Surface enhanced resonance Raman scattering (SERRS) occurs when the electronic excitation energy of the functionalized RM is close to the energy of incident light. Briefly, two types of new excited states can arise depending on the interaction among RM and substrates. As long as the energy of incident light is close to transition energies (TEs) of excited states, drastic resonance between electron transition and incident light will occur. The resonances between two different electron transitions and incident light mainly contribute to the strong enhancement of Raman signal. In addition, the EF is proportional to oscillator strength (f) of excited state, which means sufficiently large $f$ is also the precondition of intense SERRS signal. ${ }^{22}$

Compared to SERS, SERRS is far more prospective in promoting the translation of Raman imaging into clinic. First, the enhancement of SERRS is much higher than that of SERS. EF of SERS for pyridine is about $10^{6}$ whereas it goes up to $10^{15}$ in SERRS for rhodamine. The huge enhancement provides SERRS with ultra-sensitivity. Moreover, fluorescence of RM will be fully quenched on the metallic surface due to covalent interaction, which means more extensive RM could be functionalized on the substrates whether they are fluorescent or nonfluorescent. Due to the above superiorities, SERRS has showed great potential for clinical applications. By labeling the near-infrared (NIR) dyes (heptamethine cyanine dyes) on the gold nanostar, Yuan successfully detected trace NIR-SERRS probes (pM) with ultra-sensitivity. ${ }^{23}$ Additionally, other investigators also identified single molecule such as oligonucleotide, ${ }^{24}$ protein,${ }^{25}$ phospholipid $^{26}$ and thrombin ${ }^{27}$ with extremely low limit of detection $\left(<10^{-12} \mathrm{M}\right)$ with the help of 
SERRS. Owing to the much more intense Raman signal than SERS, SERRS shows great promise in tumor diagnosis and image-guided surgery. ${ }^{28,29}$ For example, pathological changes such as lymph node metastases have been successfully detected with SERRS. ${ }^{30}$

However, even though SERRS holds great promise in clinical translation, comprehensive research of the influence of substrates and RM, the two key parameters determing the sensitivity of SERRS imaging is barely studied. Heptamethine cyanine dyes possess the electronic excitation energy that is close to energy of incident laser and plasmon absorption energy of the gold nanoscaffolds in NIR wavelength window, which is a prerequisite for the occurrence of strong SERRS. In addition, the good biocompatibility makes the heptamethine cyanines safe to be used under in vivo condition. For example, indocyanine green (ICG) approved by FDA has been used in clinic for over 20 years with excellent safety record. Besides, as the most widelyused NIR fluorophores, heptamethine cyanine derivatives such as IR783 and IR780 are commercially available. Their easy accessibility offers great convenience for the investigators. As gold nanoparticle and heptamethine cyanines possess superiorities mentioned above and the heptamethine cyanines are easily resonant with gold nano-platforms in the NIR wavelength window, the combination of above materials shows potential for in vivo applications. In this work, heptamethine cyanine derivatives: IR783 and IR780 were functionalized on the three gold nanoparticle substrates, respectively. The influence of substrates'morphology (nanosphere, nanorod and nanostar) and RM (IR783 and IR780) on SERRS signal intensities were systematically studied. As shown in Fig. 1, the nanoprobes exhibited obvious
Raman signal enhancement when the RM was changed from IR783 to IR780. Considering the similar chemical structure of IR783 and IR780, the above study indicates that slight chemical structure variation of the RM could remarkably improve SERRS signal.

\section{Materials and Methods}

\subsection{Materials}

IR780 and IR783 were synthesized according to our previous work. ${ }^{31} \mathrm{HAuCl}_{4}$, sodium citrate, $\mathrm{NaBH}_{4}$, ascorbic acid, hexadecyl trimethyl ammonium bromide (CTAB), 4-(2-hydroxyethyl)-1-piperazineethanesulfonic acid (HEPES), triethylamine (TEA), AgCl, 1-(3-Dimethylaminopropyl)-3ethylcarbodiimide hydrochloride (EDC), 1-hydroxybenzotriazole (HOBT), 3-mercapropionic acid and other reagents used in the experiment were all purchased from Aladdin Chemistry (China) without further purification. The ultrapure water was from Milli-Q (Millipore, $18 \mathrm{M} \Omega$, America) source. All glassware were washed with aqua regia (hydrochloric acid: nitric acid, $3: 1 \mathrm{v} / \mathrm{v}$ ) and rinsed with ultrapure water before use.

\subsection{Synthesis and characterization}

\subsubsection{Synthesis of thiol modified IR783}

IR783 (299 mg, $0.40 \mathrm{mM}, 1.0$ eq.), $\mathrm{K}_{2} \mathrm{CO}_{3}$ (119 mg, $0.86 \mathrm{mM}, \quad 2.0$ eq. $)$ 4-carboxyphenylboronic acid (119 mg, $\quad 0.72 \mathrm{mM}, \quad 1.8$ eq.) and $\mathrm{Pd}\left[\mathrm{P}(\mathrm{Ph})_{3}\right]_{4}$ (26.6 mg, $0.020 \mathrm{mM}, 0.050$ eq.) were dissolved in $2 \mathrm{~mL}$ water, the reaction mixture was stirred at $95^{\circ} \mathrm{C}$ for $2 \mathrm{~h}$. The resulting green solid mixture was washed with cold $\mathrm{Et}_{2} \mathrm{O}$ and purified by column

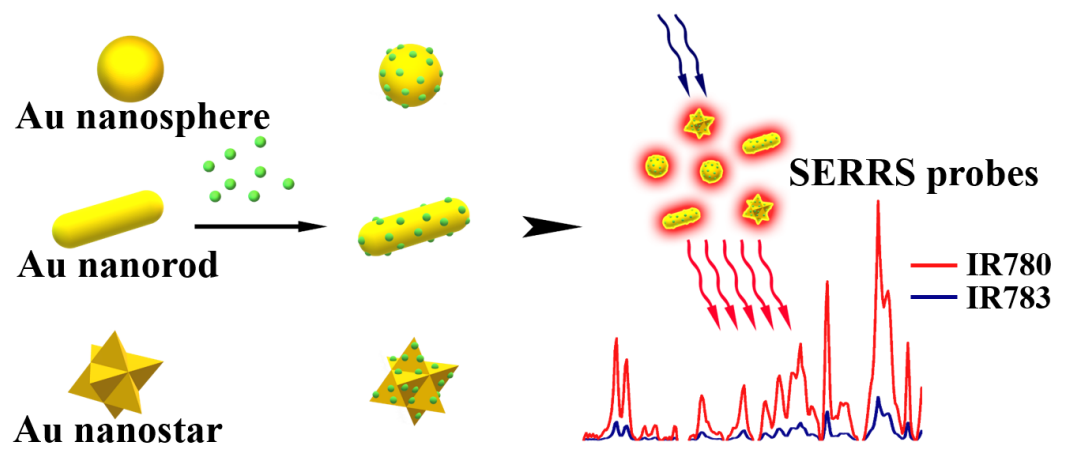

- Reporter Molecules (IR783 or IR780) Strong Enhancement

Fig. 1. Heptamethine cyanine based reporter molecule remarkably enhances SERRS signal intensity of gold nanoprobes. 
chromatography $\left(\mathrm{CH}_{2} \mathrm{Cl}_{2}: \mathrm{MeOH}=10: 3\right)$ to obtain compound 1 (292 mg, 90\%).

Compound 1 (166 mg, $0.20 \mathrm{mM}, 1.0$ eq.), EDC (3.80 mg, $0.020 \mathrm{mM}, 0.10$ eq.) and $100 \mu \mathrm{L}$ TEA were added into $6 \mathrm{~mL}$ DMF. After 5 min reaction, condensation agent HOBT (29.7 mg, $0.22 \mathrm{mM}, 1.1$ eq.) was added. Then after $10 \mathrm{~min}$ of mixing, $0.30 \mathrm{mM}$ $\beta$-Mercaptoethylamine was dissolved into the reaction system. The reaction was kept away from light for $24 \mathrm{~h}$ and the green product was purified by column chromatography $\left(\mathrm{CH}_{2} \mathrm{Cl}_{2}: \mathrm{MeOH}=10: 2.8\right)$. Final product compound $\mathbf{2}$ (146 mg, 85\%) was obtained.

\subsubsection{Synthesis of thiol modified IR 780}

To a solution of IR780 (140 mg, $0.26 \mathrm{mmol}, 1.0$ eq.) in $5 \mathrm{~mL}$ DMF 3 -mercapropionic acid $(48.9 \mu \mathrm{L}$, $0.49 \mathrm{mmol}, 1.9$ eq. $)$ and TEA $(67.9 \mu \mathrm{L}, 0.49 \mathrm{mmol}$, 1.9 eq.) were added. The green solution was stirred in the dark overnight. A green solid was isolated from the solution by precipitation with cold $\mathrm{Et}_{2} \mathrm{O}$. The crude product was purified by column chromatography $\left(\mathrm{CH}_{2} \mathrm{Cl}_{2}: \mathrm{MeOH}=50: 2.5\right)$ to obtain compound 1' (124 mg, 83\%).

Compound 1' (50.0 mg, 0.070 mM, 1.0 eq.), EDC ( $25.0 \mathrm{mg}, \quad 0.14 \mathrm{mM}, \quad 2.0$ eq.), HOBT (21.6 mg, $0.16 \mathrm{mM}, 2.3$ eq.) were added into $4 \mathrm{~mL}$ DMF. Above mixture was stirred at room temperature for $15 \mathrm{~min}$. At the end of reaction, $\beta$-Mercaptoethylamine $(10.0 \mathrm{mg}, 0.12 \mathrm{mM}, 1.7$ eq.) was added into the solution followed by stirring for overnight at room temperature. The crude product was purified by column chromatography $\left(\mathrm{CH}_{2} \mathrm{Cl}_{2}: \mathrm{MeOH}=50: 2\right)$. Final green product compound 2' (39.8 mg, 89\%) was obtained.

\subsubsection{Preparation of gold nanoparticles}

Nanorods were synthesized according to a published method. ${ }^{32}$ First, the seed solution was prepared by mixing $5 \mathrm{~mL} 0.20 \mathrm{M}$ CTAB solution with $5 \mathrm{~mL}$ $0.50 \mathrm{mM} \mathrm{HAuCl}_{4}$. To the stirred solution, $600 \mu \mathrm{L}$ icecold $0.010 \mathrm{M} \mathrm{NaBH}_{4}$ was added and the mixture was vigorously stirred for $2 \mathrm{~min}$. For the growth of nanorods, $5 \mathrm{~mL} 0.20 \mathrm{M}$ CTAB solution was added to $200 \mu \mathrm{L}$ of $4.0 \mathrm{mM} \mathrm{AgNO}_{3}$ solution at $25^{\circ} \mathrm{C}$. Next, $5 \mathrm{~mL} 1.0 \mathrm{mM} \mathrm{HAuCl}_{4}$ was added, after which $80 \mu \mathrm{L}$ of $0.079 \mathrm{M}$ antiscorbic acid was added. The dark yellow growth solution was gradually changed to colorless. Last, $12 \mu \mathrm{L}$ seed solution was added and the growth solution was left for the next $24 \mathrm{~h}$ at $30^{\circ} \mathrm{C}$.
Nanospheres were synthesized according to a classical protocol. ${ }^{33} \mathrm{HAuCl}_{4}(0.01 \%$, by weight, solution 1) and $\mathrm{Na}_{3}$-citrate (1\%, by weight, solution 2$)$ were prepared, respectively. To a boiling solution of $50 \mathrm{~mL}$ solution $1,0.5 \mathrm{~mL}$ solution 2 was added. The reaction was completed after $5 \mathrm{~min}$ of boiling.

Nanostars were synthesized by the green methodology reported by Xie et al. ${ }^{34}$ First, $0.8 \mathrm{~mL}$ $0.020 \mathrm{M} \mathrm{HAuCl}_{4}$ was added to the $100 \mathrm{~mL} \mathrm{HEPES}$ solution $(0.04 \mathrm{M}, \mathrm{pH} 7.4)$. The reaction was completed after leaving it rest for $30 \mathrm{~min}$ at $27^{\circ} \mathrm{C}$.

\subsubsection{Characterization}

The samples of gold nanostar/nanorod/nanosphere were characterized by UV-Visible spectroscopy and transmission electron microscopy (TEM). UV-Vis extinction spectra of gold nanostar/nanorod/nanosphere samples were measured using a Shimadzu UV2550 UV-Vis NIR spectrophotometer. TEM images were obtained with a transmission electron microscopy (JEOL, JEM-1400Plus, Japan.) at $100 \mathrm{kV}$.

\subsubsection{Fabrication of SERRS probes with different morphologies}

Different volumes $(1,3,10,30,50,70,100$ and $1000 \mu \mathrm{L}$ ) of $1.0 \times 10^{-4} \mathrm{M}$ IR780 and IR783 methanol solution were respectively added into $1 \mathrm{~mL}$ gold nanorod/nanosphere/nanostar solution (5.8× $\left.10^{-10} \mathrm{M}\right)$. After approximately $15 \mathrm{~min}$ of gentle shaking and standing for overnight, the fabrication was completed. The solution then was washed by centrifugation $(11,000 \mathrm{rpm}, 15 \mathrm{~min})$ in ultra-pure water for two times and finally was kept under $4{ }^{\circ} \mathrm{C}$ for storage.

\subsection{Signal intensity of the SERRS probes}

The measurement of SERRS intensity of the probes from 2.2.5 was performed with the Raman spectrometer (Ocean Optics, QE65Pro, America.) at $785 \mathrm{~nm}$ excitation wavelength in the range of Raman shift from $0 \mathrm{~cm}^{-1}$ to $3000 \mathrm{~cm}^{-1}$. The integration time was set as $100 \mathrm{~ms}$ and the laser power was $100 \mathrm{~mW}$. Removal of the noise of fluorescence background was conducted by using the software of LabSpec5 (ver. 2.02, 2010) and the final Raman spectra was obtained in the range of Raman shift from $400 \mathrm{~cm}^{-1}$ to $1500 \mathrm{~cm}^{-1}$ for analysis. 


\section{Result and Discussion}

\subsection{Synthesis of RMs and SERRS probes}

IR783 and IR780 were first modified by sulfhydrylation. The synthetic route is displayed in Fig. 2. Through the Au-S covalent interactions, IR783 and IR780 were stably conjugated to the surface of substrates, which results in corresponding SERRS probes.

\subsection{Characterization of substrates}

The TEM images of the substrates including gold nanosphere, nanorod and nanostar are shown in Fig. 3(a). They demonstrated similar diameter of approximately $50 \mathrm{~nm}$. Obviously, every nanostar consisted 4-8 branches and the aspect ratio of gold nanorod was approximately 3.1. Photospectroscopic studies of the gold nanoparticles in ultrapure water demonstrated the morphology dependent absorption. It was documented that gold nanoparticle of elongated shape like nanostar and nanorod displayed a strong longitudinal plasmon peak compared to spherical nanoparticle. ${ }^{35}$ The maximal absorption of the gold nanosphere was found at $520 \mathrm{~nm}$ with an extinction coefficient of $1.3 \times 10^{9}$ $\mathrm{M}^{-1} \mathrm{~cm}^{-1}$. In contrast, the maximal absorption of nanostar and nanorod was found in longer wavelength of around $720 \mathrm{~nm}$ but much higher extinction coefficients of $3.9 \times 10^{9} \mathrm{M}^{-1} \mathrm{~cm}^{-1} .36$ According to the Beer-Lambert law, the nearly identical concentration $\left(5.8 \times 10^{-10} \mathrm{M}\right)$ of substrates was regulated and their $\mathrm{UV}-\mathrm{V}$ is spectra are exhibited in Fig. 3(b).

\subsection{The Raman signal intensity of the SERRS probes}

\subsubsection{The RM concentration dependent Raman signal of the SERRS probes}

We first compared the Raman spectra of the SERRS probes in which IR783 and IR780 were functionalized as the RM, respectively. As shown in Fig. 4(a), when IR783 served as a RM, the strongest Raman peak emerged in $509 \mathrm{~cm}^{-1}$. Differently, the strongest peak of SERRS spectrum with IR780 as a $\mathrm{RM}$ existed in $1360 \mathrm{~cm}^{-1}$. The intensities of these two strongest peaks were quantified as functions of substrate morphology as well as the RM concentration $\left(1 \times 10^{-7}, 3 \times 10^{-7}, \quad 10^{-6}, 3 \times 10^{-6}\right.$, $4.8 \times 10^{-6}, 6.5 \times 10^{-6}, 9.1 \times 10^{-6}$ and $\left.5 \times 10^{-5} \mathrm{M}\right)$. SERRS intensity increased proportionally to RM concentration from $1 \times 10^{-7} \mathrm{M}$. The strongest Raman signals of the rod-shaped, spherical and star-shaped SERRS probes were recorded when the $\mathrm{RM}$ concentration reached $10^{-6} \mathrm{M}, 3 \times 10^{-7} \mathrm{M}$ and $10^{-6} \mathrm{M}$, respectively. After it reached the optimal concentration, the SERRS signal kept steady,

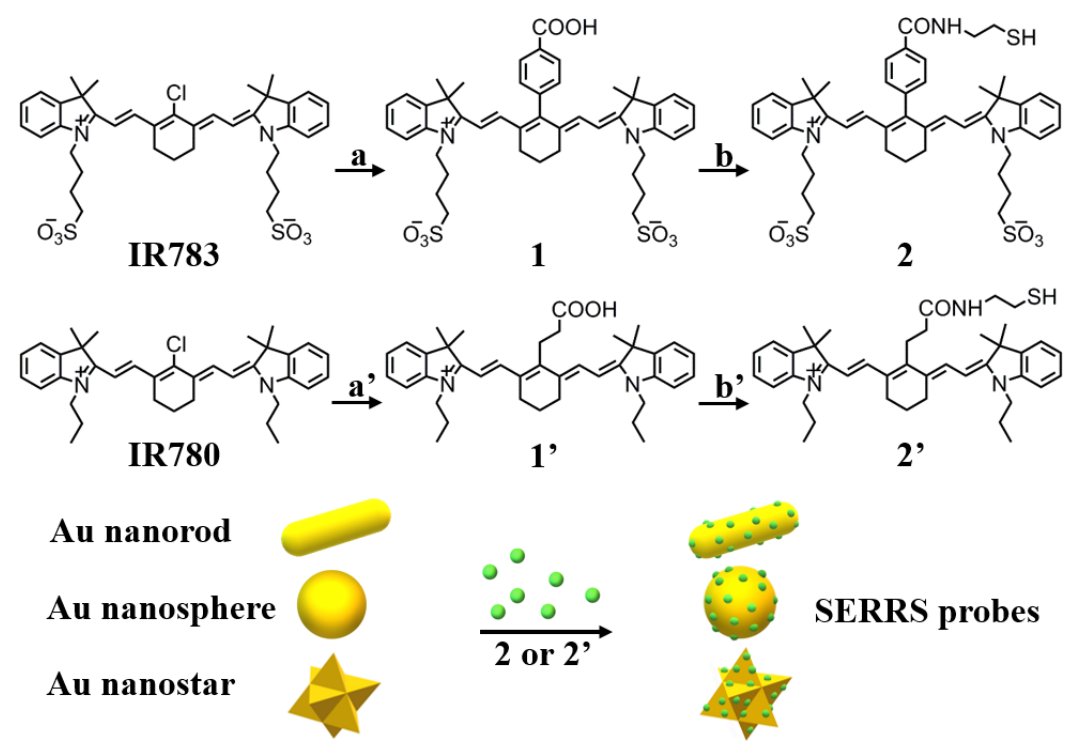

Fig. 2. Synthesis of sulfhydryl moiety functionalized RMs and SERRS nanoprobes. (a): $\mathrm{K}_{2} \mathrm{CO}_{3}$, 4-carboxyphenylboronic acid, $\mathrm{Pd}\left[\mathrm{P}(\mathrm{Ph})_{3}\right]_{4} ;(\mathrm{b})$ : TEA, EDC, HOBT, $\beta$-Mercaptoethylamine; (a'): 3-mercapropionic acid, TEA, DMF; (b'): DMF, EDC, HOBT, $\beta$-Mercaptoethylamine. 


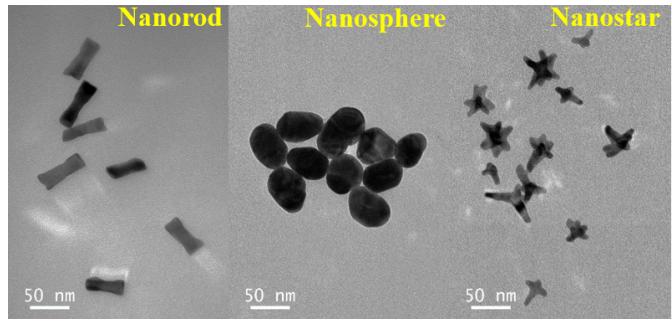

(a)

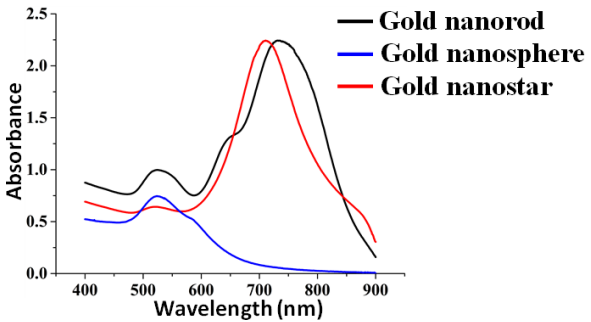

(b)

Fig. 3. Characterization of gold nanoparticle based substrates with different morphologies. (a) TEM images of gold nanorods, nanospheres and nanostars with a similar diameter. (b) The UV-Vis spectrum of the substrates with a nearly identical gold nanoparticle concentration $\left(5.8 \times 10^{-10} \mathrm{M}\right)$.

which was explained by the saturation of RM labeled on the substrate surface. Enhancement of SERRS signal by further increasing RM concentration is not recommended due to the structural instability caused by the over-crowded RM on the probe surface. According to the Avogadro's law, at the optimal concentration of $\mathrm{RM}$ for nanosphere $\left(3 \times 10^{-7} \mathrm{M}\right)$, it could be calculated that the surface of every nanosphere consisted about 520 RM molecules. Meanwhile, the optimal concentration was determined as $10^{-6} \mathrm{M}$ for both gold nanostar and nanorod, which means 1733 RM molecules were labeled on the surface of each nanorod or nanostar particle.
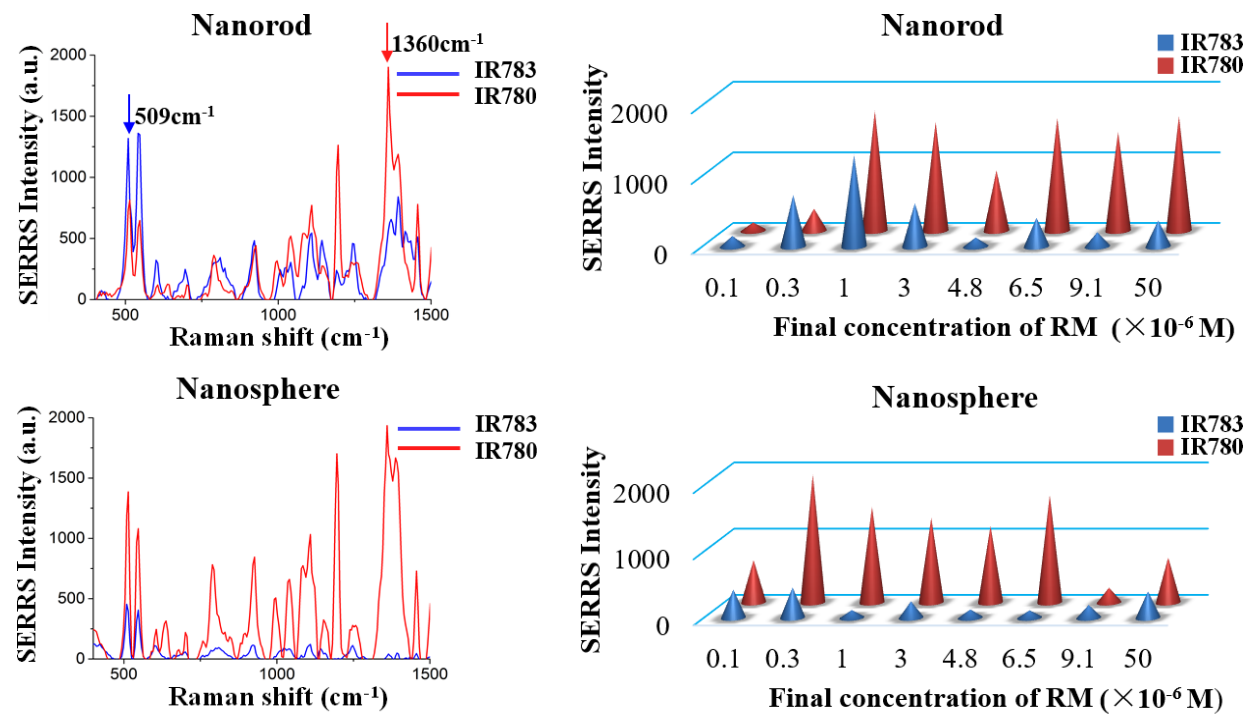

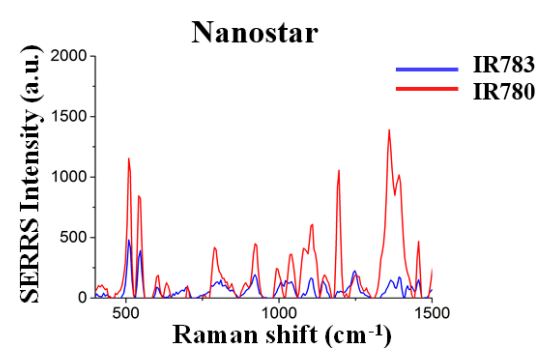

(a)

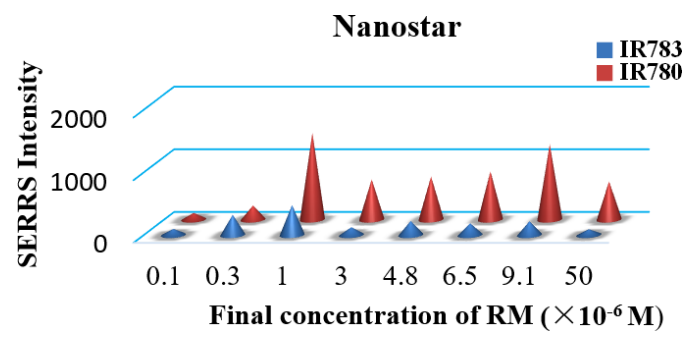

(b)

Fig. 4. The chemical structure of reporter molecule remarkably changes signal intensity of gold nanoparticle based SERRS probes. (a) The Raman spectra of the SERRS nanoprobes with different substrate morphologies and RM at their optimal concentration. (b) SERRS signal intensity of nanoprobes as a function of RM concentration and types. The Raman intensities of the gold nanoparticles were quantified from their strongest peak at $509 \mathrm{~cm}^{-1}$ (for IR783) and $1360 \mathrm{~cm}^{-1}$ (for IR780). 


\subsubsection{The substrate morphology and RM types dependent Raman signal of the SERRS probe}

Numerous works demonstrated much higher SERRS signal intensity of the nanorod and nanostar than that of nanosphere due to the enhanced "hot spot" effect. Unexpectedly, the distinction of SERRS signal intensities between the probes with different morphology was not substantial. SERRS signal of the nanorod probe only increased about 1.0 time than that of nanosphere probe when IR783 served as RM (Fig. 4(a)). Moreover, the SERRS signal from nanostar probe was roughly similar with that of nanosphere probe regardless of the RM used. Surprisingly, even though IR780 and IR783 possess very similar chemical structure, the SERRS signal generated by IR780 could reach 14 times of magnitude higher than that of IR783 when RM was $6.5 \times 10^{-6} \mathrm{M}$. Above experimental results indicated that the influence of the heptamethine cyanine based RM to the SERRS signal was remarkably greater than that of substrate morphology. Chemical structure modification of the RM could lead to more robust SERRS signal than transforming the nanoparticle substrates. The reasons may be explained as follows: (1) due to the reciprocity among the substrates and RM, the new excited states that have exclusive TE and oscillator strength (f) will emerge. Every excited state corresponds to its own SERRS peak in Raman spectrum. If the energy of incident light is coincident with or close to the TE and oscillator strength (f) is large enough, strong resonance and the intensity of corresponding SERRS peak will be enormously enhanced. In our experiment, the wavelength of excited laser was constant at $785 \mathrm{~nm}$, the variation of chemical structure of $\mathrm{RM}$ resulted in the TE which corresponds to the peak of $1360 \mathrm{~cm}^{-1}$ became closer to the energy of incident laser $(785 \mathrm{~nm})$. As a result, the degree of resonance was hugely increased which further contributed to the enormous enhancement of SERRS intensity of peak at $1360 \mathrm{~cm}^{-1}$. (2) Doering reported that halide ions $\left(\mathrm{Cl}^{-}, \mathrm{Br}^{-}\right.$, and $\left.\mathrm{I}^{-}\right)$have a substantial activating effect in chemical enhancement. ${ }^{37}$ The iodide ion in IR780 may further enhance its Raman signal. (3) The polarity of IR783 is greatly higher than IR780's due to the two sulfonic acid groups at the terminals of the side chains. The high polarity of IR783 may cause the instability of SERRS probes which attenuated the SERRS enhancement. Therefore, the optimized RM for strong SERRS intensity should possess the following characters: (1) the energy of electronic excitation is coincident with or close to that of incident laser; (2) moderate polarity; (3) possessing one or more halide ions.

\section{Conclusion}

Development of Raman probes with high sensitivity is crucial to accelerate their biomedical applications. This work indicates that slight change in the chemical structure of heptamethine cyanine based $\mathrm{RM}$ could result in remarkable enhancement of the SERRS signal. Precise modification of the RM structure can be a feasible way to develop robust SERRS probes that hold tremendous promise in accelerating their applications such as image-guided tumor resection and real-time screening biomarkers with ultralow limit of detection (LOD).

\section{Acknowledgments}

This work was financially supported by the National Basic Research Program of China (973 Program, 2013CB932500), the National Natural Science Foundation of China (Nos 81371624, 81571741), the Nanotechnology Program of Shanghai Science and Technology Committee (13NM1400400, 15140901300). The innovation and entrepreneurship training program for college students in Huazhong University of Science \& Technology (14A215). Yunfei Zhang and Danqi Li contributed equivalently to this work.

\section{References}

1. M. Fleischmann, P. J. Hendra, A. McQuillan, "Raman spectra of pyridine adsorbed at a silver electrode," Chem. Phys. Lett. 26, 163-166 (1974).

2. J. Creighton, "Surface Raman electromagnetic enhancement factors for molecules at the surface of small isolated metal spheres: The determination of adsorbate orientation from SERS relative intensities," Surf. Sci. 124, 209-219 (1983).

3. A. Campion, J. Ivanecky III, C. Child, M. Foster, "On the mechanism of chemical enhancement in surface-enhanced Raman scattering," J. Am. Chem. Soc. 117, 11807-11808 (1995). 
4. P. N. Goel, S. Singh, C. Murali Krishna, R. Gude, "Investigating the effects of Pentoxifylline on human breast cancer cells using Raman spectroscopy," J. Innov. Opt. Health Sci. 8, 1550004 (2015).

5. J. Lin, J. Lin, Z. Huang, P. Lu, J. Wang, X. Wang, R. Chen, "Raman spectroscopy of human hemoglobin for diabetes detection," J. Innov. Opt. Health Sci. 7, 79-80 (2014).

6. X. Wang, X. Qian, J. J. Beitler, Z. G. Chen, F. R. Khuri, M. M. Lewis, H. J. C. Shin, S. Nie, D. M. Shin, "Detection of circulating tumor cells in human peripheral blood using surface-enhanced Raman scattering nanoparticles," Cancer Res. 71, 15261532 (2011).

7. T. Vo-Dinh, L. R. Allain, D. L. Stokes, "Cancer gene detection using surface-enhanced Raman scattering (SERS)," J. Raman Spectrosc. 33, 511-516 (2002).

8. M. Y. Sha, H. Xu, M. J. Natan, R. Cromer, "Surface-enhanced Raman scattering tags for rapid and homogeneous detection of circulating tumor cells in the presence of human whole blood," J. Am. Chem. Soc. 130, 17214-17215 (2008).

9. X. Gao, C. Li, "Nanoprobes visualizing gliomas by crossing the blood brain tumor barrier," Small. 10, 426-440 (2014).

10. H. Yan, L. Wang, J. Wang, X. Weng, H. Lei, X. Wang, L. Jiang, J. Zhu, W. Lu, X. Wei, "Twoorder targeted brain tumor imaging by using an optical/paramagnetic nanoprobe across the blood brain barrier," ACS Nano. 6, 410-420 (2011).

11. H. Karabeber, R. Huang, P. Iacono, J. M. Samii, K. Pitter, E. C. Holland, M. F. Kircher, "Guiding brain tumor resection using surface-enhanced Raman scattering nanoparticles and a hand-held Raman scanner," ACS Nano. 8, 9755-9766 (2014).

12. P. Hildebrandt, M. Stockburger, "Surface-enhanced resonance Raman spectroscopy of Rhodamine 6G adsorbed on colloidal silver," J. Phys. Chem. 88, 5935-5944 (1984).

13. C.-G. Blatchford, J. Campbell, J. Creighton, "Plasma resonance-enhanced raman scattering by absorbates on gold colloids: The effects of aggregation," Surf. Sci. 120, 435-455 (1982).

14. H. Huang, F. Yan, Y. Kek, C. Chew, G. Xu, W. Ji, P. Oh, S. Tang, "Synthesis, characterization, and nonlinear optical properties of copper nanoparticles," Langmuir 13, 172-175 (1997).

15. E. Le Ru, M. Meyer, E. Blackie, P. Etchegoin, "Advanced aspects of electromagnetic SERS enhancement factors at a hot spot," J. Raman. Spectrosc. 39, 1127-1134 (2008).

16. C. Hrelescu, T. K. Sau, A. L. Rogach, F. Jäckel, J. Feldmann, "Single gold nanostars enhance Raman scattering," Appl. Phys. Lett. 94, 153113 (2009).
17. X.-M. Qian, S. Nie, "Single-molecule and singlenanoparticle SERS: From fundamental mechanisms to biomedical applications," Chem. Soc. Rev. 37, 912-920 (2008).

18. T. Demeritte, R. Kanchanapally, Z. Fan, A. K. Singh, D. Senapati, M. Dubey, E. Zakar, P. C. Ray, "Highly efficient SERS substrate for direct detection of explosive TNT using popcorn-shaped gold nanoparticle-functionalized SWCNT hybrid," Analyst. 137, 5041-5045 (2012).

19. J. Xie, Q. Zhang, J. Y. Lee, D. I. Wang, "The synthesis of SERS-active gold nanoflower tags for in vivo applications," ACS Nano. 2, 2473-2480 (2008).

20. J. Pérez-Juste, I. Pastoriza-Santos, L. M. LizMarzán, P. Mulvaney, "Gold nanorods: Synthesis, characterization and applications," Coordin. Chem. Rev. 249, 1870-1901 (2005).

21. J. Chen, F. Saeki, B. J. Wiley, H. Cang, M. J. Cobb, Z.-Y. Li, L. Au, H. Zhang, M. B. Kimmey, X. Li, "Gold nanocages: bioconjugation and their potential use as optical imaging contrast agents," Nano Lett. 5, 473-477 (2005).

22. M. Sun, S. Wan, Y. Liu, Y. Jia, H. Xu, "Chemical mechanism of surface-enhanced resonance Raman scattering via charge transfer in pyridine-Ag2 complex," J. Raman Spectrosc. 39, 402-408 (2008).

23. H. Yuan, Y. Liu, A. M. Fales, Y. L. Li, J. Liu, T. Vo-Dinh, "Quantitative surface-enhanced resonant Raman scattering multiplexing of biocompatible gold nanostars for in vitro and ex vivo detection," Anal. Chem. 85, 208-212 (2012).

24. K. Faulds, R. P. Barbagallo, J. T. Keer, W. E. Smith, D. Graham, "SERRS as a more sensitive technique for the detection of labelled oligonucleotides compared to fluorescence," The Analyst. 129, 567 (2004).

25. S. Habuchi, M. Cotlet, R. Gronheid, G. Dirix, J. Michiels, J. Vanderleyden, F. C. De Schryver, J. Hofkens, "Single-molecule surface enhanced resonance Raman spectroscopy of the enhanced green fluorescent protein," J. Am. Chem. Soc. 125, 84468447 (2003).

26. N. P. Pieczonka, G. Moula, R. F. Aroca, "SERRS for single-molecule detection of dye-labeled phospholipids in Langmuir-Blodgett monolayers," Langmuir. 25, 11261-11264 (2009).

27. H. Cho, B. R. Baker, S. Wachsmann-Hogiu, C. V. Pagba, T. A. Laurence, S. M. Lane, L. P. Lee, J. B.H. Tok, "Aptamer-based SERRS sensor for thrombin detection," Nano Lett. 8, 4386-4390 (2008).

28. A. M. Mohs, M. C. Mancini, S. Singhal, J. M. Provenzale, B. Leyland-Jones, M. D. Wang, S. Nie, "Hand-held spectroscopic device for in vivo and intraoperative tumor detection: Contrast 
enhancement, detection sensitivity, and tissue penetration," Anal. Chem. 82, 9058-9065 (2010).

29. G. Bodelon, V. Montes-Garcia, C. Fernandez-Lopez, I. Pastoriza-Santos, J. Perez-Juste, L. M. Liz-Marzan, "Au@pNIPAM SERRS Tags for Multiplex Immunophenotyping Cellular Receptors and Imaging Tumor Cells," Small 11, 4149-4157 (2015).

30. M. Spaliviero, S. Harmsen, R. Huang, M. A. Wall, C. Andreou, J. A. Eastham, K. A. Touijer, P. T. Scardino, M. F. Kircher, "Detection of Lymph Node Metastases with SERRS Nanoparticles," Mol. Imag. Biol. (2016).

31. L. Wang, X. Zhu, C. Xie, N. Ding, X. Weng, W. Lu, X. Wei, C. Li, "Imaging acidosis in tumors using a pH-activated near-infrared fluorescence probe," Chem. Commun. 48, 11677-11679 (2012).

32. B. Nikoobakht, M. A. El-Sayed, "Preparation and growth mechanism of gold nanorods (NRs) using seed-mediated growth method," Chem. Mater. 15, 1957-1962 (2003).
33. G. Frens, "Controlled nucleation for the regulation of the particle size in monodisperse gold suspensions," Nature 241, 20-22 (1973).

34. J. Xie, J. Y. Lee, D. I. C. Wang, "Seedless, Surfactantless, High-Yield Synthesis of Branched Gold Nanocrystals in HEPES Buffer Solution," Chem. Mater. 19, 2823-2830 (2007).

35. H. Chen, X. Kou, Z. Yang, W. Ni, J. Wang, "Shapeand size-dependent refractive index sensitivity of gold nanoparticles," Langmuir 24, 5233-5237 (2008).

36. C. J. Orendorff, C. J. Murphy, "Quantitation of metal content in the silver-assisted growth of gold nanorods," J. Phys. Chem. B 110, 3990-3994 (2006).

37. W. E. Doering, S. Nie, "Single-molecule and singlenanoparticle SERS: Examining the roles of surface active sites and chemical enhancement," J. Phys. Chem. B 106, 311-317 (2002). 